\title{
Complexes of Domain Walls in Ferromagnetic Stripes
}

\author{
A. JANUTKA* \\ Institute of Physics, Wrocław University of Technology, Wybrzeże Wyspiańskiego 27, 50-370 Wrocław, Poland \\ (Received April 16, 2013; in final form July 8, 2013)

\begin{abstract}
Interaction of domain walls in ferromagnetic stripes is studied with relevance to the formation of stable complexes of many domains. Two domain wall system is described with the Landau-Lifshitz-Gilbert equation including regimes of narrow and wide stripes which correspond the presence of transverse and vortex domain walls. The domain walls of both kinds are characterized with their chiralities (the direction of the magnetization rotation in the stripe plane) and polarities (the magnetization orientation in the center of a vortex and/or halfvortices), hence, their interactions are analyzed with dependence on these properties. In particular, pairs of the domain walls of opposite or like chiralities and polarities are investigated as well as pairs of opposite (like) chiralities and of like (opposite) polarities. Conditions of the creation of stationary bubbles built of two interacting domain walls are formulated with relevance to the situations of presence and absence of the external magnetic field.
\end{abstract}

DOI: $10.12693 /$ APhysPolA.124.641

PACS: 05.45.Yv, 75.70.Kw, 75.75.Jn, 75.78.Fg, 85.70.Kh

\section{Introduction}

Recent growth of the interest in states of many domain walls (DWs) in quasi-1D ferromagnets is due to hopes for miniaturization of information registers and logical devices based on magnetic nanowires [1-4] and nanorings [5]. Currently, the main technological effort is focused on the (straight or curved) magnetic nanostripes whose spin structure is more complex than described within the 1D idealization. It is because nanostructures of the best quality are obtained in this form using lithography methods [6]. Mutual interactions of the DWs influence the stability of a record of bits encoded in a string of the magnetic domains [4].

In the present paper, we study interactions of DWs in soft-ferromagnetic stripes of big-enough thicknesses (exceeding many times the magnetostatic exchange length as discussed in Ref. [7]) within the so-called local approach to micromagnetics (note that this magnetostatic exchange length is about $5 \mathrm{~nm}$ for permalloys). We include two regimes of the stripe widths; narrow stripes correspond to the so-called transverse DWs while wider stripes to the so-called vortex DWs [8-10]. Here and in Ref. [7], it is shown that both kinds of the DWs correspond to exact stationary solutions to the LandauLifshitz-Gilbert (LLG) equation in 2D (different realizations of the cross-tie DW [11-13]), however, their orientations in the stripe plane are distinguished by different boundary conditions. Both transverse and vortex DWs are characterized by two features; a chirality (the direction of magnetization rotation in the stripe plane) which is clockwise or counterclockwise and a polarity (the direction of magnetization alignment in a vortex/halfvortex center) which is positive or negative $[14,15]$. Pairs of the DWs of the opposite (like) both the chiralities and polarities are found to constitute exact stationary solutions to

*e-mail: Andrzej.Janutka@pwr.wroc.pl
LLG in the absence of external field, hence, they do not interact. The interactions of the DWs of opposite chiralities and of like polarities (as well as of like chiralities and of opposite polarities) are studied within a perturbation calculus previously developed with relevance to DWs in 1D ferromagnetic wire and in critical systems [16]. Evaluating the dependence of energy of the two-DW systems on the distance between the DWs, we analyze the creation of stationary bound states of two DWs (bubbles).

In the presence of an external magnetic field, even DWs of the opposite chiralities and polarities (of like chiralities and polarities) interact. The field-induced collision of such DWs is studied applying a method developed to 1D systems in [17]. For this aim, an extension of the (dissipative) evolution equation of magnetization is performed in a way to describe the dynamics in the limits of large positive and large negative values of time. The collision is found to be accompanied by the reflection of the DWs, hence, by the creation of stable bubbles of magnetization.

In Sect. 2, the single DW solutions to the LLG equation in magnetic stripes are analyzed. The interactions of the transverse and vortex DWs of opposite chiralities and of like polarities (as well as of like chiralities and of opposite polarities) are investigated within the perturbation calculus in Sect. 3 while the field-induced collisions of the DWs of opposite (like) chiralities and polarities are studied in Sect. 4. Main conclusions are summarized in Sect. 5 .

\section{Single DW in ferromagnetic stripe}

I consider stationary DW solutions to the LLG equation in $2 \mathrm{D}$

$$
\begin{gathered}
\frac{\partial \boldsymbol{m}}{\partial t}=\frac{J}{M} \boldsymbol{m} \times\left(\frac{\partial^{2} \boldsymbol{m}}{\partial x^{2}}+\frac{\partial^{2} \boldsymbol{m}}{\partial z^{2}}\right)+\gamma \boldsymbol{m} \times \boldsymbol{H} \\
+\frac{\beta_{1}}{M}(\boldsymbol{m} \cdot \hat{i}) \boldsymbol{m} \times \hat{i}-\frac{\alpha}{M} \boldsymbol{m} \times \frac{\partial \boldsymbol{m}}{\partial t} .
\end{gathered}
$$

The first term on the right hand side (r.h.s.) of (1) relates to the exchange interactions while the second (Zeeman) term depends on the external magnetic field 
$\boldsymbol{H}=\left(H_{x}, 0,0\right)$, thus, $\gamma$ denotes the gyromagnetic factor. The constant $\beta_{1}$ determines strength of the easy-axis anisotropy and $\hat{i} \equiv(1,0,0)$ indicates the long axis of the stripe. In nanomagnets with structural disorder (permalloys are the most popular materials for nanostripes), the effective spin anisotropy is dominated by dipolar interactions, hence, it is a shape dependent and mainly surface effect. Then, for stripes of big enough cross-section, the bulk anisotropy is negligible, $\beta_{1} \ll J / w^{2}$, where $w$ denotes the stripe width. Since (1) is valid only when the constraint $|m|=M$ is satisfied, one writes equations of the unconstrained dynamics equivalent to (1). Introducing $m_{ \pm} \equiv m_{y} \pm \mathrm{i} m_{z}$, one represents the magnetization components using a pair of complex functions $g(x, z, t)$, $f(x, z, t)$. The relation between the primary and secondary dynamical variables

$$
m_{+}=\frac{2 M}{f^{*} / g+g^{*} / f}, \quad m_{x}=M \frac{f^{*} / g-g^{*} / f}{f^{*} / g+g^{*} / f}
$$

ensures that $|m|=M$. Inserting (2) into (1) leads, following the Hirota method for solving nonlinear equations $[18,19]$, to the bilinear reduction of the LLG equation

$$
\begin{aligned}
& {\left[-\mathrm{i} D_{t}+J\left(D_{x}^{2}+D_{z}^{2}\right)+\alpha D_{t}\right] f^{*} \cdot g} \\
& \quad-\left(\gamma H_{x}+\beta_{1}\right) f^{*} \cdot g=0, \\
& {\left[-\mathrm{i} D_{t}-J\left(D_{x}^{2}+D_{z}^{2}\right)+\alpha D_{t}\right] f^{*} \cdot g} \\
& \quad+\left(-\gamma H_{x}+\beta_{1}\right) f^{*} \cdot g=0, \\
& \left(D_{x}^{2}+D_{z}^{2}\right) g \cdot g=0, \quad\left(D_{x}^{2}+D_{z}^{2}\right) f^{*} \cdot f^{*}=0,
\end{aligned}
$$

where $D_{t}, D_{x}, D_{z}$ denote the Hirota operators of differentiation

$$
\begin{aligned}
& D_{x}^{n} b(x, z, t) \cdot c(x, z, t) \equiv\left(\partial / \partial x-\partial / \partial x^{\prime}\right)^{n} b(x, z, t) \\
& \quad \times\left. c\left(x^{\prime}, z^{\prime}, t^{\prime}\right)\right|_{x=x^{\prime}, z=z^{\prime}, t=t^{\prime}} .
\end{aligned}
$$

For $\boldsymbol{H}=\mathbf{0}$, stationary single-DW solutions to (3) take the form

$$
f=1, \quad g=u \mathrm{e}^{k x+q z},
$$

where

$$
k^{2}+q^{2}=\frac{\beta_{1}}{J}
$$

and $\operatorname{Re} k \neq 0$. We denote $k \equiv k^{\prime}+\mathrm{i} k^{\prime \prime}, q \equiv q^{\prime}+\mathrm{i} q^{\prime \prime}$. Assuming one of the DW ends to be centered at $x=0$ (then $u=\mathrm{e}^{\mathrm{i} \varphi}$ ), the relevant magnetization profile [the single-DW solution to (1)] is written explicitly with

$$
\begin{aligned}
& m_{+}(x, z)=M \mathrm{e}^{\mathrm{i}\left(\varphi+k^{\prime \prime} x+q^{\prime \prime} z\right)} \operatorname{sech}\left(k^{\prime} x+q^{\prime} z\right), \\
& m_{x}(x, z)=-M \tanh \left(k^{\prime} x+q^{\prime} z\right) .
\end{aligned}
$$

We assume $q^{\prime \prime} \neq 0$ since, in the case $q^{\prime \prime}=0$, the DW states are similar to DWs in $1 \mathrm{D}$ and cannot exist in absence of the bulk anisotropy $[16,17,19]$. Defining $\theta \equiv$ $\arctan \left(q^{\prime} / k^{\prime}\right)$, following (5), one finds $k^{\prime \prime}=-q^{\prime \prime} \tan (\theta)$ and $k^{\prime 2}-q^{\prime \prime 2}=\beta_{1} /\left\{J\left[1+\tan ^{2}(\theta)\right]\right\}$. Also, we assume the magnetization alignments on both the stripe edges to be similar, the ordering is symmetric with respect to the line $z=w / 2$. It leads to the condition $k^{\prime \prime}\left(-w q^{\prime} / k^{\prime}\right)+q^{\prime \prime} w=n \pi$ where $n=1,2, \ldots$ and, finally, to $q^{\prime \prime}=n \pi /\left\{w\left[1+\tan ^{2}(\theta)\right]\right\}$.
Additional boundary condition is related to minimization of the surface (magnetostatic) energy and it discriminates between different values of $\varphi, n$, and $\theta$. We evaluate the dependence of the energy of the DW on these parameters using the Hamiltonian $\mathcal{H}=\mathcal{H}_{0}+\mathcal{H}_{Z}$, where

$$
\begin{aligned}
& \mathcal{H}_{0}=\frac{J}{2 M}\left(\left|\frac{\partial \boldsymbol{m}}{\partial x}\right|^{2}+\left|\frac{\partial \boldsymbol{m}}{\partial z}\right|^{2}\right)+\frac{\beta_{1}}{2 M}\left[M^{2}-(\boldsymbol{m} \cdot \hat{i})^{2}\right], \\
& \mathcal{H}_{Z}=-\gamma \boldsymbol{H} \cdot \boldsymbol{m}
\end{aligned}
$$

$\left(\mathcal{H}_{Z}\right.$ denotes its Zeeman part). Total energy of the DW $E=E_{0}+E_{Z}+E_{\mathrm{B}}$ is the sum of the bulk energy $E_{0}+E_{Z}$, defined by $E_{0(Z)} \equiv \int_{-\infty}^{\infty} \int_{0}^{w} \mathcal{H}_{0(Z)} \mathrm{d} z \mathrm{~d} x$, and of the boundary energy $E_{\mathrm{B}}$. The contribution $E_{\mathrm{B}}$ is determined referring to a theorem by Carbou who proved that the magnetostatic energy of any ferromagnetic element of finite thickness $\tau ; 1 / \lambda^{2} \int_{S}(\boldsymbol{m} \cdot \boldsymbol{n})^{2} \mathrm{~d} s$ tends to $\left(1 / \Lambda_{2}\right) \int_{\partial S}\left(\boldsymbol{m} \cdot \boldsymbol{n}^{\prime}\right)^{2} \mathrm{~d} l$ with $\tau \rightarrow 0[20]$. Here, $S$ denotes the surface of the bulk ferromagnet and $\partial S$ denotes the boundary of the base of its solid, $n$ is normal to the magnet surface, $n^{\prime}$ denotes the unitary vector outward to the line of the base boundary. The coefficient $\Lambda_{2}$ scales with $\tau$ and with a diameter $w$ following $\Lambda_{2} \sim \lambda^{2} /[\tau|\log (\tau / w)|]$, (with relevance to a stripe, $w$ represents its width $[9,21]$ ). Writing the formula for $E_{\mathrm{B}}$, one has to notice that the Carbou theorem is not strictly applicable to systems with open boundaries (infinite stripes), however, it shows some tendency in ordering at the stripe edges. In particular, it indicates that the magnetostatic interactions in flat magnets induce more than one hard directions of magnetization parallel to the main plane. We propose to effectively describe the magnetostatic energy in the form of an integral over the stripe edge

$$
E_{\mathrm{B}}=\int_{-\infty}^{\infty}\left[-\frac{2}{\Lambda_{1}}\left(M^{2}-m_{x}^{2}\right)+\frac{2}{\Lambda_{2}} m_{z}^{2}\right]_{z=0} \mathrm{~d} x
$$

where, in contrast to [21], the effect of anisotropy relating to the long axis of the stripe is included via the term depending on $\Lambda_{1}$. By analogy to systems with boundaries of finite length that satisfy the Carbou theorem, this coefficient is expected to scale with the stripe width following $\Lambda_{1} \sim \lambda^{2} / w$. More detailed estimation of $E_{\mathrm{B}}$ is performed in Appendix A and in Ref. [7].

Inserting (6) into the Hamiltonian (7), one arrives at

$$
E_{0}(\theta, n)=2 J M w \sqrt{\frac{\beta_{1}}{J}\left[1+\tan ^{2}(\theta)\right]+\frac{\pi^{2} n^{2}}{w^{2}}} .
$$

Estimating $E_{\mathrm{B}}$, we divide it into two parts $E_{\mathrm{B}}=E_{\mathrm{B} 1}+$ $E_{\mathrm{B} 2}$;

$$
\begin{aligned}
& E_{\mathrm{B} 1}(\theta, n) \equiv-\frac{2 M^{2}}{\Lambda_{1}} \int_{-\infty}^{\infty} \operatorname{sech}^{2}\left(\frac{n \pi x}{w\left[1+\tan ^{2}(\theta)\right]}\right) \mathrm{d} x, \\
& E_{\mathrm{B} 2}(\varphi, \theta, n) \equiv \frac{2 M^{2}}{\Lambda_{2}} \int_{-\infty}^{\infty} \sin ^{2}\left(\varphi-\frac{n \pi \tan (\theta) x}{w\left[1+\tan ^{2}(\theta)\right]}\right) \\
& \quad \times \operatorname{sech}^{2}\left(\frac{n \pi x}{w\left[1+\tan ^{2}(\theta)\right]}\right) \mathrm{d} x .
\end{aligned}
$$


a)
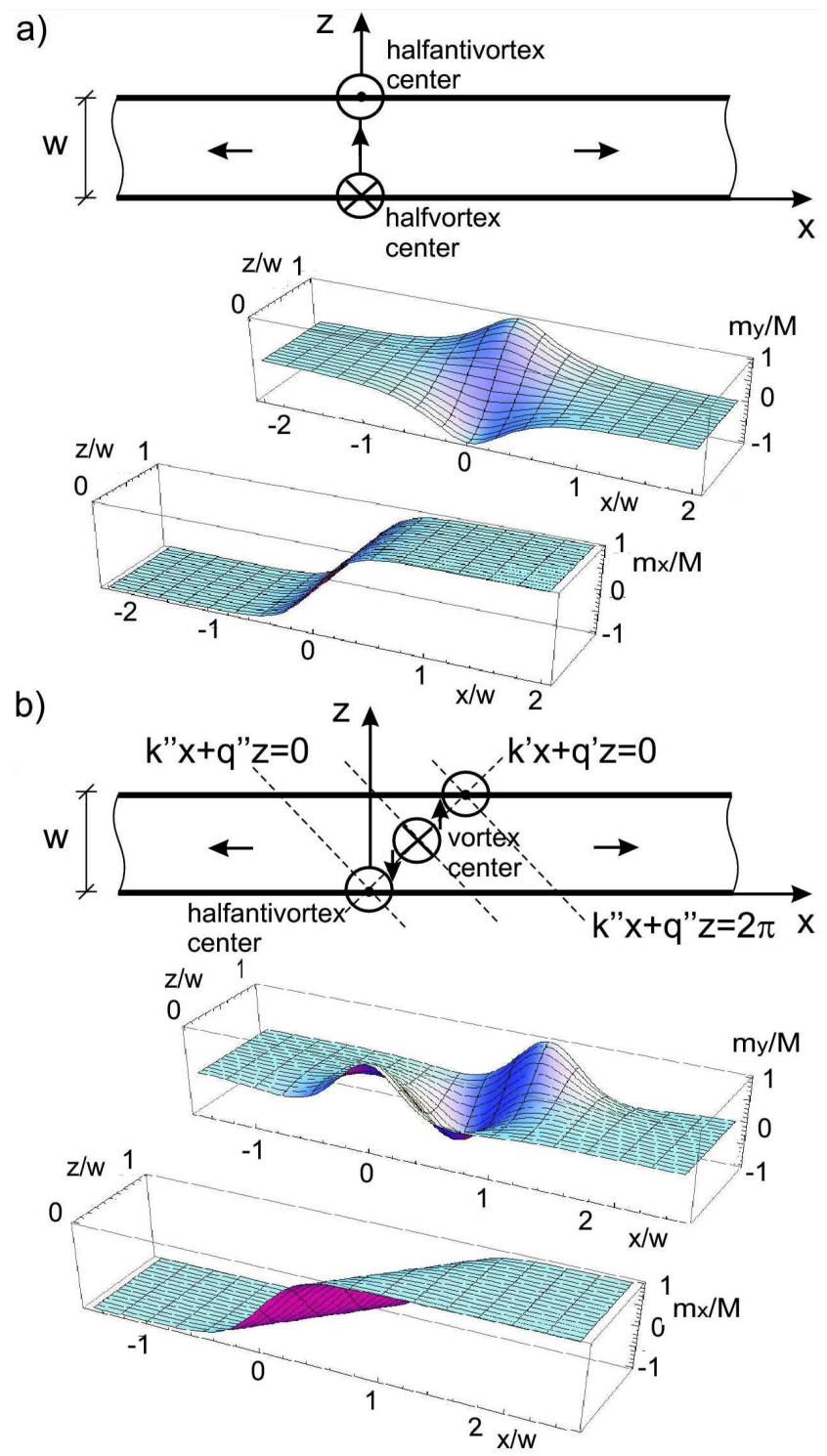

Fig. 1. DW configurations: (a) a transverse DW, (b) a vortex DW. In the upper draws, arrows indicate magnetization alignment.

The minimization of $E_{\mathrm{B} 2}(\varphi, \theta, n)$ leads to $\varphi=0, \pi$ independently of other parameters of the DW. It corresponds to the presence of halfvortices (halfantivortices) at the stripe edges (as shown in Fig. 1). In the regime of narrow stripes $w / \tau \approx 1, \Lambda_{2} \ll \Lambda_{1}\left(1 / \Lambda_{2}\right.$ is small, thus, $E_{0}$ dominates over $\left.E_{\mathrm{B}}\right)$, via minimization of $E_{0}(\theta, n)$ the smallest possible value of $n$ is preferable while the minimization of both the energy components $E_{0}(\theta, n), E_{\mathrm{B}}(0, \theta, n)$ leads to the preferred values of the angle $\theta=0, \pi$. Increase of the stripe width $w$ with fixed thickness $\tau$ results in the transition to the regime $\Lambda_{2}>\Lambda_{1}$. Furthermore, as mentioned previously, for soft-magnetic alloys, $\beta_{1}$ is negligibly small, $\beta_{1} \ll J / w^{2}$, thus, from (9), $E_{0}(\theta, n)=E_{0}(n)$ is independent of $w$, and $E_{\mathrm{B}}$ becomes comparable to $E_{0}$. For this case, via the minimization of
$E_{\mathrm{B} 1}(\theta, n)$, the biggest possible value of $\tan ^{2}(\theta)$ and the smallest value of $n$ are preferable, whereas, for $\theta \neq 0, \pi$, the minimization of $E_{\mathrm{B} 2}(0, \theta, n)$ indicates big values of $n$ to be preferable. Hence, the transition from the DW state of $n=1, \theta=0, \pi$ to a state of $n=2, \theta \neq 0$ takes place with increase of $w$. For $n=2$, the condition $\tan ^{2}(\theta)>1$ has to be satisfied in order to $E_{\mathrm{B}}(0, \theta, 2)<E_{\mathrm{B}}(0,0,1)$.

The state $n=1$ and $\theta=0, \pi$ corresponds to $k^{\prime \prime}=q^{\prime}=$ $0,\left|q^{\prime \prime}\right|=\pi / w,\left|k^{\prime}\right|=\sqrt{\pi^{2} / w^{2}+\beta_{1} / J}$ and it is called a transverse $D W$. With relevance to the state $n=2$, we take $\beta_{1}=0$ (the shape anisotropy does not affect the dynamical equation while it is completely included in the boundary condition, the minimization of $E_{\mathrm{B}}$ ), and $|\theta|$ to be close to its infimum $|\theta| \approx \pi / 4$. The resulting magnetization structure corresponds to $\left|q^{\prime}\right| \approx\left|q^{\prime \prime}\right| \approx\left|k^{\prime}\right| \approx\left|k^{\prime \prime}\right| \approx$ $\pi / w$ and one calls it a vortex $D W$. The polarity of vortex (transverse) DW (the magnetization orientation in the center of vortex/halfvortex, parallel or antiparallel) is determined by value of $\varphi$ while $\operatorname{sgn}\left(q^{\prime \prime}\right)$ determines its chirality (the magnetization rotation in the stripe plane, clockwise or anticlockwise to $y$ axis).

\section{Interaction of static domain walls}

Let us analyze the interaction of transverse (vortex) DWs within a perturbation calculus developed earlier with relevance to the interactions of DWs in 1D ferromagnet [16]. In the present section, we focus on the systems whose interacting DWs are of opposite chiralities and of like polarities (or of like chiralities and of opposite polarities) visualized in Fig. 2. we refer to the remaining cases (the DWs of opposite chiralities and polarities as well as the DWs of like chiralities and polarities) in the next section.
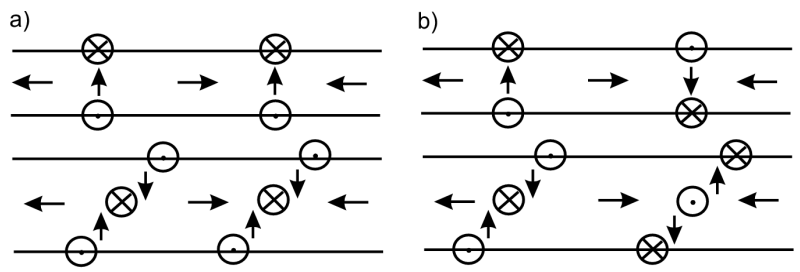

Fig. 2. Scheme of the magnetization layout in double-DW configurations: (a) pair of transverse (parallel vortex) DWs of opposite chiralities and of like polarities, (b) pair of transverse (parallel vortex) DWs of like chiralities and of opposite polarities.

Locally, in the vicinity of the center of $j$-th DW $(j=$ $1,2)$, one can write the magnetization in the form

$$
\boldsymbol{m}(x, z, 0)=\boldsymbol{m}^{(j)}(x, z)+\delta \boldsymbol{m}^{(j)}(x, z),
$$

where $\boldsymbol{m}^{(j)}$ denotes the stationary single-DW solution to $(1)$ :

$$
\begin{aligned}
& m_{+}^{(j)}(x, z)=M \mathrm{e}^{\mathrm{i}\left\{\varphi_{j}+\delta_{j}\left[k^{\prime \prime}\left(x-x_{0 j}\right)+q^{\prime \prime} z\right]\right\}} \\
& \quad \times \operatorname{sech}\left(\sigma_{j}\left[k^{\prime}\left(x-x_{0 j}\right)+q^{\prime} z\right]\right), \\
& m_{x}^{(j)}(x, z)=-M \tanh \left(\sigma_{j}\left[k^{\prime}\left(x-x_{0 j}\right)+q^{\prime} z\right]\right),
\end{aligned}
$$

while $\delta \boldsymbol{m}^{(j)}$ denotes a perturbation due to the presence 
of another DW. Here $\delta_{1}=\delta_{2}= \pm 1,\left|\sigma_{j}\right|=1, \sigma_{1}=-\sigma_{2}$, and, for $k^{\prime}>0, \sigma_{j}=1$ corresponds to DW of the head-to-head type while $\sigma_{j}=-1$ to the tail-to-tail type. The phases satisfy $\varphi_{1}=\varphi_{2}$ or $\varphi_{1}=\varphi_{2} \pm \pi$. We refer to the case $\delta_{1}=\delta_{2}$ and $\varphi_{1}=\varphi_{2}$ as to the pair of walls of opposite chiralities and of like polarities while to the case $\delta_{1}=\delta_{2}$ and $\varphi_{1}=\varphi_{2} \pm \pi$ as to the walls of like chiralities and of opposite polarities. In terms of vortex DWs, we focus our attention on the interaction of mutually parallel vortex DWs while taking the single DWs profile in the form (12) excludes its use to non-parallel DWs. The non-parallel vortex walls overlap at one edge of the stripe much stronger than at the second edge, thus, an effective description of their interaction can be performed with 1D model of Ref. [16] applied to the stripe-edge magnetization. In order to fulfill the constraint $|\boldsymbol{m}|=M$, we take the perturbation of an initially static DW state in the form

$$
\begin{gathered}
\delta \boldsymbol{m}^{(j)}=\left( \pm \frac{m_{x}^{(k)}}{M}-1\right) \boldsymbol{m}^{(j)} \pm \frac{m_{x}^{(j)}}{M}\left(0, m_{y}^{(k)}, m_{z}^{(k)}\right) \\
\mp \frac{1}{M}\left(m_{y}^{(k)} m_{y}^{(j)}+m_{z}^{(j)} m_{z}^{(k)}, 0,0\right),
\end{gathered}
$$

where $k \neq j$, which leads to

$$
\begin{aligned}
& m_{x}= \pm \frac{1}{M}\left[m_{x}^{(1)} m_{x}^{(2)}-\frac{1}{2}\left(m_{+}^{(1)} m_{-}^{(2)}+m_{+}^{(2)} m_{-}^{(1)}\right)\right], \\
& m_{+}= \pm \frac{1}{M}\left(m_{+}^{(1)} m_{x}^{(2)}+m_{+}^{(2)} m_{x}^{(1)}\right) .
\end{aligned}
$$

The plus and minus relate to the bubble magnetization parallel and antiparallel to $x$-axis, respectively. We stress that (14) is irrelevant to the case of DWs of opposite (like) chiralities and polarities, $\delta_{1}=-\delta_{2}$, since, for this case, $|m|=M$ was not satisfied. In order to satisfy $|m|=M$, the product $m_{+}^{(1)} m_{-}^{(2)}$ should take real values. Moreover, with relevance to vortex DWs, this condition is fulfilled for discrete values of $a=n \pi$ only, where $n$ is an integer. The perturbation of states of the interacting DWs should be small. According to (13), this condition is fulfilled when the interacting DWs are far-enough from each other [functions of the r.h.s. of (13) satisfy $\left| \pm m_{x}^{(k)} / M-1\right|,\left|m_{y}^{(k)} / M\right|,\left|m_{z}^{(k)} / M\right| \ll 1$ if the distance between the walls is larger than their width].

With relevance to the transverse DWs, insertion of (14), with $\left|q^{\prime}\right|=\left|k^{\prime \prime}\right|=0$, into the Hamiltonian (7) and integration over the stripe area $\{x \in(-\infty, \infty)$, $z \in[0, w]\}$ leads to the following dependence of the energy of a temporal static state of the DW pair on the distance of separation of the walls

$$
\begin{aligned}
& E_{0}(a)=\frac{M}{2} w J k^{\prime} I^{ \pm}(a, 0) \\
& =\frac{M}{2} w \sqrt{J \beta_{1}+\frac{J^{2} \pi^{2}}{w^{2}}} \operatorname{csch}^{2 \mp 1}(a / 2) \\
& \times \operatorname{sech}^{2 \pm 1}(a / 2)[-2 a+\sinh (2 a)]
\end{aligned}
$$

where $a \equiv k^{\prime}\left(x_{02}-x_{01}\right)$, and $I^{ \pm}(a, \theta)$ represent integrals given in Appendix B. The upper signs correspond to the pair of transverse DWs of opposite chiralities and of like polarities, the case $\varphi_{1}=\varphi_{2}$, while the lower signs correspond to the pair of transverse DWs of like chiralities and of opposite polarities, the case $\varphi_{1}=\varphi_{2} \pm \pi$. Up to a multiplicative constant, the above energy dependences on $a$ are similar to the ones of the pairs of DWs (of opposite chiralities and of like chiralities, respectively) in $1 \mathrm{D}$ ferromagnet [16].

For parallel vortex DWs, inserting (14), with $\left|q^{\prime\left({ }^{\prime \prime}\right)}\right|=$ $\left|k^{\prime\left({ }^{\prime \prime}\right)}\right|=\pi / w$ and $\beta_{1}=0$, into the Hamiltonian and integrating it over the stripe area, one arrives at

$$
\begin{aligned}
& E_{0}(a)=M w J k^{\prime} I^{ \pm}(a, 1) \\
& =\pi M J \frac{1}{6} \operatorname{csch}^{5}(a)\{[36 a \cosh (a)-12 a \cosh (3 a) \\
& \quad-24 \sinh (a)-5 \sinh (3 a)+3 \sinh (5 a)] \\
& \mp 24 \cos (a) \sinh ^{2}(a)[-2 a+\sinh (2 a)] \\
& -\cos (2 a)[24 a \cosh (a)-18 \sinh (a)-2 \sinh (3 a)]\},
\end{aligned}
$$

where upper signs correspond to the pair of DWs of opposite chiralities and of like polarities while lower signs to the pair of DWs of like chiralities and of opposite polarities. The energy dependences on the DW separation distance $(15),(16)$ are plotted in Fig. 3a and b, respectively. Extrema of $E_{0}(a)+E_{Z}(a)$ correspond to stationary states of DW pairs; stable (minima) or unstable (maxima) ones. The character of extremum (minimum or maximum) of the function $E_{0}(a)$ at $a=0$ indicates that the interaction of the (transverse or vortex) DWs of opposite chiralities and like polarities is attractive when their separation distance is short while the interaction of DWs of like chiralities and opposite polarities is repulsive in this case. To be precise, here, we call the distance between DWs short when it is close to their width since the perturbation calculus is applicable to $|a|>1$.
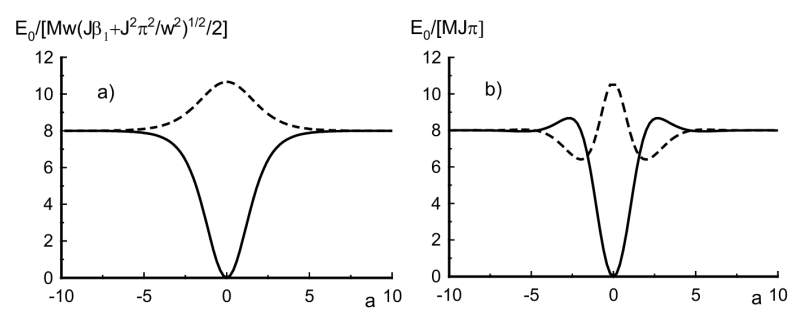

Fig. 3. Energy of a pair of DWs with dependence on the distance of their separation: (a) transverse DWs, (b) vortex DWs. Solid line - DWs of opposite chiralities and of like polarities, dotted line - DWs of like chiralities and of opposite polarities.

In the case of presence of an external longitudinal field $H_{x} \neq 0$, the energy dependence on the distance of DW separation $E_{0}(a)+E_{Z}(a)$ deviates from these in Fig. 3a and $\mathrm{b}$ due to non-zero Zeeman term, however, such a deviation is small for $a$ close to zero, similar to one in Fig. 1 of Ref. [16]. Moreover, the Zeeman term is arbitrarily 
small when restrict considerations to weak-enough external field. With this restriction, due to attractive interaction, a (field-induced) collision of transverse or vortex DWs of opposite chiralities and of like polarities is expected to result in their mutual annihilation, according to simulations for the transverse DWs [22, 23]. Correspondingly, due to repulsive interaction, the collision of transverse or vortex DWs of like chiralities and of opposite polarities leads to their mutual reflection. Both the DW annihilation and the bubble nucleation have been observed in ferromagnetic nanorings [24, 25].

Unlike in the case of transverse DWs, the interaction of vortex DWs changes its character from attractive to repulsive (or vice versa) with increasing the distance between the DWs.

\section{Field-induced collision of domain walls}

In the present section, the interaction of DWs of opposite chiralities and polarities (as well as of like chiralities and polarities) is analyzed. It is shown that such pairs of DWs create static bubbles of magnetization which are exact stationary solutions to the LLG equation. Hence, the walls do not interact in absence of an external field. The application of a magnetic field in the opposite direction to the bubble magnetization induces motion of both the DWs towards each other and, eventually, their collision. It is accompanied by an interaction of the DWs due to a dynamical deformation of them which cannot be described in the framework of the above used perturbation calculus.

Stationary two-DW solutions to (1) have been found in the absence of anisotropy, for $\beta_{1}=0$. Under this condition, for $\boldsymbol{H}=0$, inserting the ansatz $f=1$, $g=u \mathrm{e}^{k_{1} x+q_{1} y} \pm u \mathrm{e}^{k_{2} x+q_{2} y}$ into (3), one finds the relations

$$
k_{1(2)}^{2}+q_{1(2)}^{2}=0, \quad k_{1} k_{2}+q_{1} q_{2}=0 .
$$

Let $k_{j} \equiv k_{j}^{\prime}+\mathrm{i} k_{j}^{\prime \prime}, q_{j} \equiv q_{j}^{\prime}+\mathrm{i} q_{j}^{\prime \prime}$. For double-DW solutions, $k_{1}^{\prime}=-k_{2}^{\prime}= \pm \pi / w$, the above conditions lead to $q_{1}^{\prime \prime}=-q_{2}^{\prime \prime},\left|q_{1(2)}^{\prime \prime}\right|=\pi / w, k_{1(2)}^{\prime \prime}=q_{1(2)}^{\prime}=0$ for transverse DWs, and to $k_{1}^{\prime} k_{2}^{\prime \prime}=k_{1}^{\prime \prime} k_{2}^{\prime}, q_{1}^{\prime} q_{2}^{\prime \prime}=q_{1}^{\prime \prime} q_{2}^{\prime}$, $\left|k_{1(2)}^{\prime \prime}\right|=\left|q_{1(2)}^{\prime}\right|=\left|q_{1(2)}^{\prime \prime}\right|=\pi / w$ for vortex DWs.

Concerning nonstationary DW solutions to the LLG equation for $\boldsymbol{H} \neq 0$ and $\alpha \neq 0$, we notice that solitary-wave solutions to the LLG equation are relevant in the limit of large positive values of time only, whenever magnetostatic effects which lead to the Walker breakdown (an easy-plane anisotropy in the case of $1 \mathrm{D}$ ferromagnet) are included [26-28]. Hence, studying the dynamics of a pair of DWs which are stationary at the initial moment, one cannot avoid an effect of non-adiabatic switching the magnetic field on. On the other hand, standard approach to the problem of soliton collision is based on the analysis of asymptotics of two soliton solutions in the limits $t \rightarrow \pm \infty$, e.g. it has been used with relevance to collisions of spontaneously propagating topological solitons (DWs) in 1D ferromagnet (in absence of external field and dissipation) [18]. In the presence of dissipation this method fails since solutions to dissipative equations of motion become unphysical in the limit of large negative values of time, because of divergence of energy in this limit. In particular, the Zeeman energy of any ferromagnetic stripe diverges with $t \rightarrow-\infty$ whereas domains magnetized parallel (antiparallel) to the magnetic field grow (diminish) with time. In view of the purpose of studying field-induced collisions, these facts motivate extension of the dynamical system (3) within a formalism applicable to the limits of large positive and negative values of time.

The method of extension of the dynamical system has been developed in Ref. [17]. It is based on a Bateman idea of doubling the number of degrees of freedom when including the dissipation into the standard Lagrange formalism (with relevance to damped harmonic oscillator) [29]. We change secondary dynamical Eqs. (3) replacing $g, g^{*}, f, f^{*}$ with novel fields of the corresponding set $g_{1}, g_{2}^{*}, f_{2}, f_{1}^{*}$ and of the set of their c.c. in a way that the resulting extended system of the equations of motion

$$
\begin{aligned}
& \alpha D_{t} f_{1}^{*} \cdot g_{1}-\gamma H_{x} f_{1}^{*} \cdot g_{1}=\mathrm{i} D_{t} f_{1}^{*} \cdot g_{1}, \\
& \left(D_{x}^{2}+D_{z}^{2}\right) f_{1}^{*} \cdot g_{1}=0, \\
& \left(D_{x}^{2}+D_{z}^{2}\right) g_{1} \cdot g_{1}=0, \quad\left(D_{x}^{2}+D_{z}^{2}\right) f_{1}^{*} \cdot f_{1}^{*}=0
\end{aligned}
$$

and of their conjugates [that differ from (18) by the sign of the dissipation constant $\alpha$ ]

$$
\begin{aligned}
& -\alpha D_{t} f_{2}^{*} \cdot g_{2}-\gamma H_{x} f_{2}^{*} \cdot g_{2}=\mathrm{i} D_{t} f_{2}^{*} \cdot g_{2}, \\
& \left(D_{x}^{2}+D_{z}^{2}\right) f_{2}^{*} \cdot g_{2}=0, \\
& \left(D_{x}^{2}+D_{z}^{2}\right) g_{2} \cdot g_{2}=0, \quad\left(D_{x}^{2}+D_{z}^{2}\right) f_{2}^{*} \cdot f_{2}^{*}=0
\end{aligned}
$$

is, in a formal sense, symmetric with respect to the reversal of the arrow of time (although, dissipative dynamics of any physical system is irreversible). Comparing (18) and (19), one sees that $g_{2}(x, z, t)\left[f_{2}(x, z, t)\right]$ can be obtained from $g_{1}(x, z, t)\left[f_{1}(x, z, t)\right]$ via changing the sign of its parameter $\alpha$. Upon the change $t \rightarrow-t$, the system of the novel equations transforms into itself if one accompanies this operation by the transform of the novel dynamical variables $g_{1(2)} \rightarrow f_{2(1)}, f_{1(2)} \rightarrow-g_{2(1)}$. The above trick is an analogous construction to classical and quantum formalisms for description of dissipative systems in the whole length of the time axis despite the divergence of excitation energy in the limit of large negative values of time (non-equilibrium Green functions, thermo-field dynamics, rigged Hilbert space) [30], which are all based on the concept of Bateman. Equations (18) and their c.c. determine the magnetization dynamics for large positive values of time (in particular, for $t \rightarrow \infty$ ). Therefore, the magnetization vector should be expressed with the functions $g_{1}, g_{1}^{*}, f_{1}, f_{1}^{*}$ in the relevant time regime. Writing the magnetization in the form

$$
m_{+}=\frac{2 M}{f_{1}^{*} / g_{1}+g_{1}^{*} / f_{1}}, \quad m_{x}=M \frac{f_{1}^{*} / g_{1}-g_{1}^{*} / f_{1}}{f_{1}^{*} / g_{1}+g_{1}^{*} / f_{1}}
$$

ensures that their components satisfy $|\boldsymbol{m}|=M, m_{x}=$ $m_{x}^{*}$, and they reproduce (2) for $\alpha=0$. In the limit $t \rightarrow-\infty$, similar to the quantum field theory approach, the response of the system is determined with novel fields. In this limit, one can analyze the evolution of the mag- 
netization with vector $\tilde{\boldsymbol{m}}$ defined as

$$
\tilde{m}_{+}=-\frac{2 M}{f_{2}^{*} / g_{2}+g_{2}^{*} / f_{2}}, \quad \tilde{m}_{x}=-M \frac{f_{2}^{*} / g_{2}-g_{2}^{*} / f_{2}}{f_{2}^{*} / g_{2}+g_{2}^{*} / f_{2}} .
$$

The single-DW solution to (18), (19) is of the forn $f_{2}^{*}=\mathrm{e}^{-l t / 2}, g_{1}=u \mathrm{e}^{k x+q z-l t / 2}$, which leads to the magnetization profile

$$
\begin{aligned}
& m_{+}(x, z, t)=M \mathrm{e}^{\mathrm{i}\left[\varphi+k^{\prime \prime}\left(x-x_{0}\right)+q^{\prime \prime} z-l^{\prime \prime} t\right]} \\
& \quad \times \operatorname{sech}\left(k^{\prime}\left(x-x_{0}\right)+q^{\prime} z-l^{\prime} t\right), \\
& m_{x}(x, z, t)=-M \tanh \left(k^{\prime}\left(x-x_{0}\right)+q^{\prime} z-l^{\prime} t\right),
\end{aligned}
$$

and represents the translationally and rotationally moving transverse DW of $\left|k^{\prime}\right|=\left|q^{\prime \prime}\right|=\pi / w,\left|k^{\prime \prime}\right|=\left|q^{\prime}\right|=0$ or

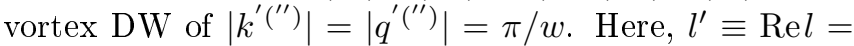
$\gamma H_{x} \alpha /\left(1+\alpha^{2}\right), l^{\prime \prime} \equiv \operatorname{Im} l=\gamma H_{x} /\left(1+\alpha^{2}\right)$. The nonzero $l^{\prime \prime}$ corresponds to the DW evolution in strong enough magnetic field that exceeds significantly the Walker breakdown value $\left|H_{x}\right| \gg H_{\mathrm{W}}[31,32]$. Below the Walker breakdown, in a weak external field, the rotation of the magnetization about $x$ axis is suppressed by the minimization of surface energy (a strong effective biaxial anisotropy [33]). The relevant dynamics is described with a differing from (1) primary evolution equation and differing from (18), (19) secondary equations which are obtained by replacing the l.h.s. of (1) and r.h.s. of (18), (19) with zero $[7,17]$. In this weak-field regime, $l^{\prime}=\gamma H_{x} / \alpha$, $l^{\prime \prime}=0$. In the intermediate region, above the Walker breakdown $\left|H_{x}\right|>H_{\mathrm{W}}$, the field-induced motion of the DW is accompanied by a dynamic transformation of its structure [34]. The DW oscillatory changes between the transverse and vortex ones [35]. My further considerations focus on the regime $\left|H_{x}\right|<H_{\mathrm{W}}$, then the motion of a single DW is a simple translation.

For $H \neq 0$, two-DW solution to modified (by taking their l.h.s. equal to zero) Eqs. (18), (19) takes the form

$$
\begin{aligned}
& f_{1}^{*}=\mathrm{e}^{\gamma H_{x} t /(2 \alpha)}, \\
& g_{1}=u\left(\mathrm{e}^{k_{1} x+q_{1} z} \pm \mathrm{e}^{k_{2} x+q_{2} z}\right) \mathrm{e}^{-\gamma H_{x} t /(2 \alpha)}, \\
& f_{2}^{*}=\mathrm{e}^{-\gamma H_{x} t /(2 \alpha)}, \\
& g_{2}=u\left(\mathrm{e}^{k_{1} x+q_{1} z} \pm \mathrm{e}^{k_{2} x+q_{2} z}\right) \mathrm{e}^{\gamma H_{x} t /(2 \alpha)},
\end{aligned}
$$

where plus (minus) corresponds to states of a pair of the DWs of like (opposite) chiralities and polarities, while the parameters $k_{1(2)}, q_{1(2)}$ satisfy the conditions (17). Hence, for $k_{1}^{\prime}=-k_{2}^{\prime}$ and $q_{1}^{\prime}=-q_{2}^{\prime}$ (parallel DWs); $k_{1}^{\prime \prime}=-k_{2}^{\prime \prime}$ and $q_{1}^{\prime \prime}=-q_{2}^{\prime \prime}$. It should be emphasized that the above single-DW and double-DW solutions to (18) satisfy the original system (3).

Let

$$
\begin{aligned}
& \eta_{j}(x, z, t) \equiv \sigma_{j} \pi / w\left(x-x_{0 j}+\theta z\right)-\gamma H_{x} t / \alpha, \\
& \tilde{\eta}_{j}(x, z, t) \equiv \sigma_{j} \pi / w\left(x-x_{0 j}+\theta z\right)+\gamma H_{x} t / \alpha, \\
& \xi_{j}(x, z) \equiv \delta_{j} \pi / w\left[\theta\left(x-x_{0 j}\right)-z\right],
\end{aligned}
$$

with $\sigma_{1}=-\sigma_{2}=1, \delta_{1}=-\delta_{2},\left|\delta_{1(2)}\right|=1$, and $\theta=0$ for transverse DWs while $\theta=1$ for vortex DWs. For $H_{x}>0$ and $\eta_{k} \ll \eta_{j} \approx 0$, here $k \neq j(k, j=1,2)$, we find the distant-future limit of the magnetization (20)

$$
\lim _{t \rightarrow \infty} m_{+}=m_{+}^{(j)} \equiv( \pm 1)^{j-1} 2 M \frac{v \mathrm{e}^{\eta_{j}} \mathrm{e}^{\mathrm{i} \xi_{j}}}{1+\mathrm{e}^{2 \eta_{j}}},
$$

$$
\lim _{t \rightarrow \infty} m_{x}=m_{x}^{(j)} \equiv M \frac{1-\mathrm{e}^{2 \eta_{j}}}{1+\mathrm{e}^{2 \eta_{j}}} .
$$

Identifying the parameters $x_{0 j}$ with the DW-center positions $x_{01}=-x_{02}\left[\ln (u) \propto \sigma_{1}+\mathrm{i} \delta_{1} \theta=-\sigma_{2}-\mathrm{i} \delta_{2} \theta\right]$, We introduce the restriction on $v ; v=1$ or $v=-1$. The magnetization profiles (25) describe the motion of well separated DWs of the type presented with (22) and correspond to the limit $t \rightarrow \infty$ of the DW solutions to the LLG equation in 2D for $\left|H_{x}\right|<H_{\mathrm{W}}$ by Slonczewski $[28,36]$.

In the distant-past limit, we describe the magnetization evolution using the field $\tilde{\boldsymbol{m}}$. Following (21), for $\tilde{\eta}_{j} \ll \tilde{\eta}_{k} \approx 0$ and $j \neq k$,

$$
\begin{aligned}
& \lim _{t \rightarrow-\infty} \tilde{m}_{+}=\tilde{m}_{+}^{(j)} \equiv-( \pm 1)^{k-1} 2 M \frac{v \mathrm{e}^{\tilde{\eta}_{k}} \mathrm{e}^{\mathrm{i} \xi_{k}}}{1+\mathrm{e}^{2 \tilde{\eta}_{k}}}, \\
& \lim _{t \rightarrow-\infty} \tilde{m}_{x}=\tilde{m}_{x}^{(j)} \equiv-M \frac{1-\mathrm{e}^{2 \eta_{k}}}{1+\mathrm{e}^{2 \tilde{\eta}_{k}}} .
\end{aligned}
$$

Considering the collision of DWs which are infinitely distant from each other at the beginning of their evolution, we determine the magnetization dynamic in the limit $t \rightarrow-\infty$, via inverting the propagation direction of the kinks of $\tilde{\boldsymbol{m}}$ and reversing the arrow's head of the field vector $\tilde{\boldsymbol{m}}$. Utilizing the properties $\tilde{m}_{+}^{(j)}(x+$ $\left.x_{0 k}, z, 0\right) \mathrm{e}^{\mathrm{i} \xi_{k}(-x,-z)}=\tilde{m}_{+}^{(j)}\left(-x+x_{0 k},-z, 0\right) \mathrm{e}^{\mathrm{i} \xi_{k}(x, z)}$, $\tilde{m}_{x}^{(j)}\left(x+x_{0 k}, z, 0\right)=-\tilde{m}_{x}^{(j)}\left(-x+x_{0 k},-z, 0\right)$, for $\eta_{j} \gg$ $\eta_{k} \approx 0,(j \neq k)$, we arrive at

$$
\begin{aligned}
& m_{+}(x, z, t)=-\tilde{m}_{+}^{(j)}\left(-x+2 x_{0 k},-z, t\right) \mathrm{e}^{\mathrm{i} 2 \xi_{k}(x, z)}, \\
& m_{x}(x, z, t)=-\tilde{m}_{x}^{(j)}\left(-x+2 x_{0 k},-z, t\right) .
\end{aligned}
$$

The applicability of the above procedure to the asymptotic evolution of a single DW can be verified noticing that any single-DW solution to (18), (19), [Eq. (22)] satisfies

$$
\begin{aligned}
& m_{+}(x, z, t)=-\tilde{m}_{+}\left(-x+2 x_{0},-z, t\right) \mathrm{e}^{\mathrm{i} 2 \xi(x, z)}, \\
& m_{x}(x, z, t)=\tilde{m}_{x}\left(-x+2 x_{0},-z, t\right) .
\end{aligned}
$$

According to (25), (27a), (27b), two initially closing up DWs of opposite (like) chiralities and polarities have to diverge after the collision. The one of the colliding DWs that was initially, for $t \rightarrow-\infty$, described with the field ingredient $\tilde{m}_{+}^{(j)}, \tilde{m}_{x}^{(j)}$, is finally, for $t \rightarrow \infty$, described with the field ingredient $m_{+}^{(j)}, m_{x}^{(j)}$. Hence, during the collision, DWs exchange their parameters $x_{01} \leftrightarrow x_{02}$ and their phase factors. The DWs reflect in a way that one can say they pass through each other without changing their velocities and polarities, however, with changing their character from the head-to-head one into the tail-to-tail one and vice versa and their chiralities (the process is illustrated in Fig. 4). This prediction corresponds to the result of the field-induced collision of a Bloch DW with a Néel DW in 1D ferromagnet [17], as well as of the collision of spontaneously propagating (in absence of the dissipation) topological solitons (DWs) in ferromagnets which are intermediate structures between the Bloch and Néel DWs [19]. 

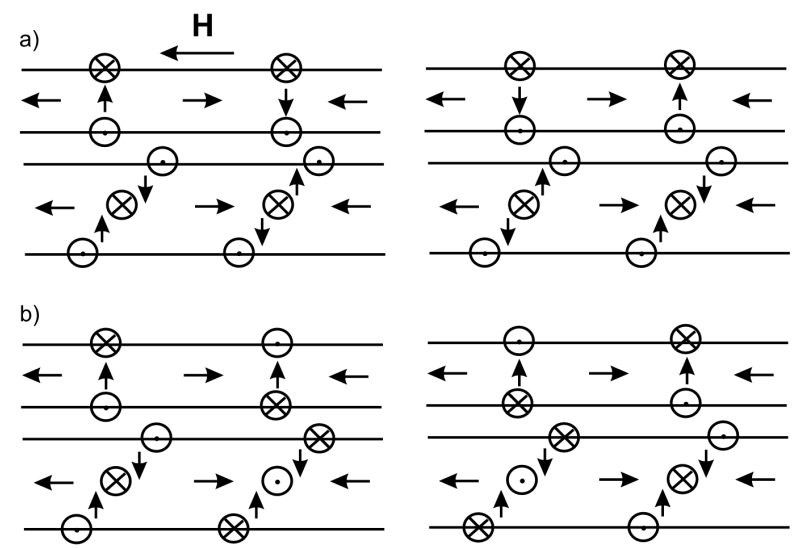

Fig. 4. Collision of DWs forming a hard magnetization bubble in a stripe. Scheme of the magnetization layout in the initial (final) state is shown in the left (right) picture for (a) pairs of transverse and vortex DWs of like chiralities and polarities, (b) pairs of transverse and vortex DWs of opposite chiralities and polarities.

We have considered systems of infinite domains whose energy cannot be defined, however, the smaller a domain is the bigger percentage of the Zeeman part of its energy is lost per time unit due to the DW motion. The field-induced DW reflection induces the motion which contradicts the rule of energy minimization. Such a motion has to be decelerated and, eventually, suppressed when the decrease of the DW interaction energy equals the increase of the Zeeman energy. It results in formation of a stationary bubble which is a counterpart of hard bubbles in $1 \mathrm{D}$ systems [17].

\section{Conclusions}

In terms of the application of multi-DW systems to the magnetic storage, the important result of the present study is the prediction of bound states of DWs [which are of opposite (like) chiralities and polarities] in the absence of any external field. Such bound states are stable with respect to simultaneous change of both the chirality and polarity of one of the DWs in the bubble. Unlike stationary bubbles in $1 \mathrm{D}$ ferromagnets which are composed of one Néel DW and one Bloch DW, the bubbles in magnetic stripes are composed of DWs of the same energy, hence, they can be stable. In 1D systems, the instability is a simple consequence of the fact that the Néel DWs are of higher energy than the Bloch ones and they tend to the reorientation into the Bloch walls in presence of fluctuations [37]. The possibility of maintaining stable train of many DWs in magnetic stripes without external power supply (without application of the magnetic field) makes such systems potentially useful as magnetic information registers.

The result of the field-induced collision of DWs of opposite chiralities and polarities (or of like chiralities and polarities) is found to be their reflection. Hence, they can form a stripe counterpart of the hard bubbles of magnetization of wide ferromagnetic platelets.
Another finding to be stressed is the existence of stationary bound state of two vortex DWs of like chiralities and of opposite polarities (a $2 \pi$-DW) in the absence of any external field. It follows from Fig. $3 \mathrm{~b}$ that the energy of the relevant pair of vortex DWs $E_{0}(a)$ achieves minima at $a \neq 0$. They correspond to the final state of the long-term evolution of a breather. Such a state has no counterpart in 1D ferromagnet while it has a counterpart in critical media described with Ginzburg-Landau (or a nonlinear Schrödinger) equation [16].

\section{Acknowledgments}

This work was supported by Polish Government Research Funds for 2010-2012 in the framework of grant No. N N202 198039.

\section{Appendix A: Estimation of boundary energy}

The magnetostatic energy of a magnetic element contains contributions that relate to interactions of surface charges, volume charges, and interaction between surface and volume ones

$$
\begin{gathered}
E_{\mathrm{MS}}=\iint \frac{\rho(\boldsymbol{x}) \rho\left(\boldsymbol{x}^{\prime}\right)}{\left|\boldsymbol{x}-\boldsymbol{x}^{\prime}\right|} \mathrm{d} V(\boldsymbol{x}) \mathrm{d} V\left(\boldsymbol{x}^{\prime}\right) \\
+\iint \frac{\sigma(\boldsymbol{x}) \sigma\left(\boldsymbol{x}^{\prime}\right)}{\left|\boldsymbol{x}-\boldsymbol{x}^{\prime}\right|} \mathrm{d} S(\boldsymbol{x}) \mathrm{d} S\left(\boldsymbol{x}^{\prime}\right) \\
+\iint \frac{\sigma(\boldsymbol{x}) \rho\left(\boldsymbol{x}^{\prime}\right)}{\left|\boldsymbol{x}-\boldsymbol{x}^{\prime}\right|} \mathrm{d} S(\boldsymbol{x}) \mathrm{d} V\left(\boldsymbol{x}^{\prime}\right),
\end{gathered}
$$

where $\rho=-\nabla \cdot \boldsymbol{m}, \sigma=\boldsymbol{n} \cdot \boldsymbol{m}$. Following Ref. [38], reducing one of the spatial dimensions with relevance to flat systems of thickness $\tau$ and neglecting volume and base-surface terms, the above expression is transformed into the energy of the boundary of $2 \mathrm{D}$ system (up to the multiplier $\tau$ )

$$
\begin{aligned}
& \tau E_{\mathrm{B}}=\tau^{2} \int_{\partial S} \int_{\partial S} \sigma(\boldsymbol{x}) \sigma\left(\boldsymbol{x}^{\prime}\right) \ln \left(\left|\boldsymbol{x}-\boldsymbol{x}^{\prime}\right| / \tau\right) \mathrm{d} l(\boldsymbol{x}) \mathrm{d} l\left(\boldsymbol{x}^{\prime}\right) \\
& \quad+\tau^{2} \int_{\partial S} \int_{S_{\text {base }}}\left[\sigma(\boldsymbol{x}) \rho\left(\boldsymbol{x}^{\prime}\right)+\rho(\boldsymbol{x}) \sigma\left(\boldsymbol{x}^{\prime}\right)\right] \\
& \quad \times \ln \left(\left|\boldsymbol{x}-\boldsymbol{x}^{\prime}\right| / \tau\right) \mathrm{d} l(\boldsymbol{x}) \mathrm{d} S\left(\boldsymbol{x}^{\prime}\right) .
\end{aligned}
$$

Here $S_{\text {base }}$ denotes the surface of the platelet base. For any DW in the stripe, $\rho(\boldsymbol{x}) \sim-\partial m_{x} / \partial x \sim\left[M^{2}-\right.$ $\left.m_{x}^{2}\right] /(M \delta)$ with $\delta$ denoting the DW width. We estimate the boundary energy with

$$
\begin{aligned}
& \tau E_{\mathrm{B}}=\tau^{2} \int_{\partial S} \int_{\partial S}\left(\boldsymbol{n}^{\prime} \cdot \boldsymbol{m}\right)(\boldsymbol{x})\left(\boldsymbol{n}^{\prime} \cdot \boldsymbol{m}\right)\left(\boldsymbol{x}^{\prime}\right) \\
& \quad \times \ln \left(\left|\boldsymbol{x}-\boldsymbol{x}^{\prime}\right| / \tau\right) \mathrm{d} l(\boldsymbol{x}) \mathrm{d} l\left(\boldsymbol{x}^{\prime}\right) \\
& \quad-\tau^{2} \int_{\partial S} \int_{S_{\mathrm{base}}}\left(\boldsymbol{n}^{\prime} \cdot \boldsymbol{m}\right)(\boldsymbol{x}) \frac{\partial m_{x}}{\partial x}\left(\boldsymbol{x}^{\prime}\right) \\
& \quad \times \ln \left(\left|\boldsymbol{x}-\boldsymbol{x}^{\prime}\right| / \tau\right) \mathrm{d} l(\boldsymbol{x}) \mathrm{d} S\left(\boldsymbol{x}^{\prime}\right) \\
& \quad \approx 2 \delta \tau^{2} \ln (\delta / \tau) \int_{-\infty}^{\infty} m_{z}^{2}(x, 0,0) \mathrm{d} x
\end{aligned}
$$




$$
-2 a w \tau^{2} \ln (\delta / \tau) \int_{-\infty}^{\infty}\left[M^{2}-m_{x}^{2}(x, 0,0)\right] \mathrm{d} x,
$$

where $a w$ corresponds to an effective thickness of the surface layer of the stripe edge over which the magnetization is independent of normal coordinate $z(a \ll 1$ and $a \propto \delta)$. Since $\delta \sim w$, one arrives at $E_{\mathrm{B}}$ of $(8)$.

\section{Appendix B: Explicit form of integrals}

$$
\begin{aligned}
I^{ \pm} & (a, \theta) \equiv \int_{-\infty}^{\infty}\left(\left\{\operatorname{sech}^{2}(y) \tanh (-y+a)\right.\right. \\
& -\operatorname{sech}^{2}(-y+a) \tanh (y) \mp \cos (\theta a)[-\tanh (y) \\
& +\tanh (-y+a)] \operatorname{sech}(y) \operatorname{sech}(-y+a)\}^{2} \\
& +\{-\operatorname{sech}(y)[\tanh (y) \tanh (-y+a) \\
& \left.+\operatorname{sech}^{2}(-y+a)\right] \pm \cos (\theta a) \operatorname{sech}(-y+a) \\
& \left.\times\left[\tanh ^{2}(y) \tanh (-y+a)+\operatorname{sech}(y)\right]\right\}^{2} \\
& +\left\{\sin ^{2}(\theta a) \operatorname{sech}(-y+a)[\tanh (y) \tanh (-y+a)\right. \\
& \left.\left.+\operatorname{sech}^{2}(y)\right]\right\}^{2}+[\sin (\theta a) \tanh (y) \operatorname{sech}(-y+a)]^{2} \\
& +[\operatorname{sech}(y) \tanh (-y+a) \pm \cos (\theta a) \tanh (y) \\
& \left.\times \operatorname{sech}(-y+a)]^{2}\right) \mathrm{d} y .
\end{aligned}
$$

\section{References}

[1] S.S.P. Parkin, M. Hayashi, L. Thomas, Science 320, 190 (2008).

[2] M. Hayashi, L. Thomas, R. Moriya, C. Rettner, S.S.P. Parkin, Science 320, 209 (2008).

[3] D.A. Allwood, G. Xiong, C.C. Faulkner, D. Atkinson, D. Petit, R.P. Cowburn, Science 309, 1688 (2005).

[4] G. Hrkac, J. Dean, D.A. Allwood, Philos. Trans. R. Soc. A 369, 3214 (2011).

[5] C.B. Muratov, V.V. Osipov, IEEE Trans. Magn. 45, 3207 (2009).

[6] M. Klaui, J. Phys. Condens. Matter 20, 313001 (2008).

[7] A. Janutka, Phys. Rev. B 85, 184421 (2012); A. Janutka, IEEE Trans. Magn. 48, 3140 (2012).

[8] R.D. McMichael, M.J. Donahue, IEEE Trans. Magn. 33, 4167 (1997).

[9] O. Tchernyshyov, G.-W. Chern, Phys. Rev. Lett. 95, 197204 (2005).

[10] H. Min, R.D. McMichael, M.J. Donahue, J. Miltat, M.D. Stiles, Phys. Rev. Lett. 104, 217201 (2010).

[11] S. Middelhoek, J. Appl. Phys. 34, 1054 (1963).

[12] K.L. Metlov, Appl. Phys. Lett. 79, 2609 (2001); K.L. Metlov, J. Low. Temp. Phys. 139, 207 (2005).

[13] W.C. Uhlig, M.J. Donahue, D.T. Pierce, J. Unguris, J. Appl. Phys. 105, 103902 (2009).

[14] R. Moriya, L. Thomas, M. Hayashi, Y.B. Bazaliy, C. Rettner, S.S.P. Parkin, Nature Phys. 4, 368 (2008).

[15] A. Vanhaverbeke, A. Bischof, R. Allenspach, Phys. Rev. Lett. 101, 107202 (2008).
[16] A. Janutka, Acta Phys. Pol. A 124, 23 (2013).

[17] A. Janutka, Phys. Rev. E 83, 056607 (2011).

[18] M.M. Bogdan, A.S. Kovalev, JETP Lett. 31, 424 (1980); R. Hirota, J. Phys. Soc. Jpn. 51, 323 (1982).

[19] A.M. Kosevich, B.A. Ivanov, A.S. Kovalev, Phys. Rep. 194, 117 (1990); M. Svendsen, H. Fogedby, J. Phys. A 26, 1717 (1993).

[20] G. Carbou, Math. Models Meth. Appl. Sci. 11, 1529 (2001); R.V. Kohn, V.V. Slastikov, Arch. Rational Mech. Anal. 178, 227 (2005).

[21] G.-W. Chern, D. Clarke, H. Youk, O. Tchernyshyov, in: Quantum Magnetism, Eds. B. Barbara, Y Imry, G. Sawatzky, P.C.E. Stamp, NATO Science for Peace and Security Series B: Physics and Biophysics, Springer, Berlin 2008, p. 35.

[22] A. Kunz, Appl. Phys. Lett. 94, 132502 (2009).

[23] D. Djuhana, H.-G. Piao, S.-C. Yu, S.K. Oh, D.-H. Kim, J. Appl. Phys. 106, 103926 (2009).

[24] J. Rothman, M. Klaui, L. Lopez-Diaz, C.A.F. Vaz, A. Bleloch, J.A.C. Bland, Z. Cui, R. Speaks, Phys. Rev. Lett. 86, 1098 (2001).

[25] F.J. Castano, C.A. Ross, C. Frandsen, A. Eilez, D. Gil, H.I. Smith, M. Redjdal, F.B. Humphrey, Phys. Rev. B 67, 184425 (2003).

[26] Z. Li, S. Zhang, Phys. Rev. Lett. 92, 207203 (2004); S. Zhang, Z. Li, Phys. Rev. Lett. 93, 127204 (2004).

[27] Y. Tserkovnyak, A. Brataas, G.E.W. Bauer, J. Magn. Magn. Mater. 320, 1282 (2008).

[28] J.C. Slonczewski, J. Appl. Phys. 44, 1759 (1973).

[29] H. Bateman, Phys. Rev. 38, 815 (1931); H. Dekker, Phys. Rep. 80, 1 (1981); D. Chruscinski, J. Jurkowski, Ann. Phys. 321, 854 (2006).

[30] L.V. Keldysh, Sov. Phys. JETP 20, 1018 (1965); I. Hardman, H. Umezawa, Y. Yamanaka, J. Math. Phys. 28, 2925 (1987); I.E. Antoniou, M. Gadella, E. Karpov, I. Prigogine, G. Pronko, Chaos Solit. Fract. 12, 2757 (2001).

[31] A.P. Malozemoff, J.C. Slonczewski, Phys. Rev. Lett. 29, 952 (1972); N.L. Schryer, L.R. Walker, J. Appl. Phys. 45, 5406 (1974); A. Thiaville, J.M. Garcia J. Miltat, J. Magn. Magn. Mater. 242-245, 1061 (2002).

[32] A. Thiaville, Y. Nakatani, J. Miltat, Y. Suzuki, Europhys. Lett. 69, 990 (2005).

[33] A. Mougin, M. Cormier, J.P. Adam, P.J. Metaxas, J. Ferre, Europhys. Lett. 78, 57007 (2007).

[34] M. Hayashi, L. Thomas, C. Rettner, R. Moriya, S.S.P. Parkin, Appl. Phys. Lett. 92, 112510 (2008).

[35] J.-Y. Lee, K.-S. Lee, S. Choi, K.Y. Guslienko, S.-K. Kim, Phys. Rev. B 76, 184408 (2007); J.-Y. Lee, K.-S. Lee, S.-K. Kim, Appl. Phys. Lett. 91, 122513 (2007).

[36] J.C. Slonczewski, J. Appl. Phys. 45, 2705 (1974).

[37] J. Lajzerowicz, J.J. Niez, J. Phys. Lett. (France) 40, L165 (1979).

[38] W.F. Brown, A.E. LaBonte, J. Appl. Phys. 36, 1380 (1965); A. Aharoni, IEEE Trans. Magn. 27, 3539 (1991). 